\title{
FRECUENCIA DE ENTEROPARASITOSIS EN JARDINES INFANTILES ALEDAÑOS A LA CUENCA BAJA DEL RÍO TUNJUELITO
}

\section{ENTEROPARASITISM PREVALENCE IN PRE-SCHOLAR NURSERIES LOCATED NEAR THE LOW BASIS OF THE TUNJUELITO RIVER}

\author{
Diego M. Luna', Leonardo Camacho', Diana Rojas², Martín A. Bayona
}

\begin{abstract}
${ }^{1}$ Médico Interno, Facultad de Medicina. Universidad de Ciencias Aplicadas y Ambientales U.D.C.A. E-mail:leonardomed88@ hotmail.com. ${ }^{2}$ M.D. Especialista Epidemiología. Facultad de Medicina, Universidad de Ciencias Aplicadas y Ambientales U.D.C.A. E-mail: dianarojasa@gmail.com. ${ }^{3}$ Bacteriólogo, Especialista, M.Sc. Microbiología. Facultad de Medicina, Universidad de Ciencias Aplicadas y Ambientales U.D.C.A. Calle 222 No. 55-37, Bogotá, D.C. E-mail: mabayona@udca. edu.co, autor para correspondencia.
\end{abstract}

Rev. U.D.C.A Act. \& Div. Cient. 13 (1): 7-15, 2010

\section{RESUMEN}

El propósito del presente trabajo correspondió en determinar la frecuencia de enteroparasitosis en relación con las variables demográficas y socio-económicas, en los jardines infantiles aledaños a la cuenca baja del río Tunjuelito (Bogotá, D.C. - Colombia). Se realizó un estudio de tipo observacional descriptivo de corte transversal, entre agosto y septiembre de 2009. Se registraron datos demográficos y factores socio-económicos, se recolectaron muestras seriadas de materia fecal a 92 niños y niñas, en edades comprendidas entre 2 y 5 años, las cuales, se procesaron con métodos coproparasitológicos. Se determinó una frecuencia del $75 \%$ de parásitos intestinales, donde el $57,6 \%$ eran comensales intestinales y el 17,4, patógenos intestinales. Se encontró Giardia lamblia (7,6\%) y Entamoeba histolytica (7,6\%), como patógenos más frecuentes, seguido de Ascaris lumbricoides (2,2\%). El contacto del agua del río, el estrato social y financiero, las características del piso de la vivienda, el almacenamiento de basura y el caminar descalzo fueron las cinco variables que tuvieron relación, estadísticamente significativa, con el resultado parasitológico. Se concluye, que existen condiciones en la población estudiada, que constituyen un contexto favorable para la elevada existencia de las enfermedades parasitarias intestinales.
Palabras clave: Abundancia parásitos, Ascaris, materia fecal, factores demográficos, factores socio-económicos, población infantil.

\section{SUMMARY}

The purpose of this research was to determine the relationship between the frequency of entero-parasitism and the demographic and the socio-economic variables within pre-primary school nurseries located near the low basin of the Tunjuelito river (Bogotá, D.C., Colombia), between august and september 2009. A study of the observational, descriptive and transverse type was realized. Demographic data and socio-economic factors were registered through the application of an instrument designed by the authors. Seriated samples of fecal material from 92 children, both males and females, were collected and processed by copro-parasitological methods. A frequency of $75 \%$ of intestinal parasites was determined; $57.6 \%$ were intestine commensals and $17.4 \%$ intestinal pathogens. As most frequent pathogens Giardia lamblia $7.6 \%$ and Entamoeba histolytica $7.6 \%$ were detected, followed by Ascaris lumbricoides (2.2\%). The contact with the water of the river, the socioeconomic level, the houses floor characteristics, the waste accumulation and the barefoot walking were the five variables, statistically significant in relation to the 
parasitic results. The existence of conditions suffered by the studied population constitutes a favorable context for the elevated existence of the parasitic intestinal diseases.

Key words: Prevalence, entero-parasitosis, demographic factors, socio-economic factors, pre-primary scholars.

\section{INTRODUCCIÓN}

Las infecciones por parásitos intestinales constituyen un importante problema de salud, por sus altas tasas de prevalencia y amplia distribución mundial, sobre todo, en las regiones tropicales y subtropicales (Morrone et al. 2004). La epidemiología de las entero-parasitosis posee un componente determinante ambiental; la presencia y la transmisión efectiva de un parásito es consecuencia de un entorno que le resulta favorable (Milano et al. 2007).

Las parasitosis intestinales son infecciones muy frecuentes (prevalencia del 40-70\%) en los países subdesarrollados, debido a que en ellos coexisten malas condiciones higiénicas, escasa cultura médica, deficiente saneamiento ambiental y bajas condiciones socioeconómicas (Marcos et al. 2003; Agudelo et al. 2008). Aquellas producidas por protozoos y helmintos afectan a más de dos billones de personas de la población mundial (Morrone et al. 2004).

En Colombia, la prevalencia de parásitos es del $12 \%$ en la población general y del $28 \%$ entre niños de uno y cuatro años (Savioli et al. 1992). Según el Instituto Nacional de Salud, en la Investigación Nacional de Morbilidad realizada en 1980, se estableció que el $81,8 \%$ de las personas en el país se encontraban parasitadas; de éstos, el $63 \%$ con parásitos patógenos y $18 \%$ con parásitos no patógenos (Corredor $\mathcal{E}$ Arciniegas, 2002). Los parásitos intestinales patógenos más frecuentemente hallados en el país son Ascaris lumbricoides, Trichuris trichuria, Giardia intestinalis, Entamoeba histolytica, Enterobius vermicularis, Necator americanus, Ancylostoma duodenale, Taenia solium y T. saginata (Botero $\mathcal{E}$ Restrepo, 2003).

La Investigación Nacional de Morbilidad realizada en Colombia entre 1965 y 1980 reportó el comportamiento de los parásitos patógenos de la siguiente manera: $A$. lumbricoides, en 1965 , se encontraba en un $54 \%$ y disminuyó, en 1980, a un 34\%; T. trichiura de un 50\% reportado en 1965, en 1980 disminuyó a un 37\%; el complejo E. histolytica/dispar, en 1965, se reportó con una prevalencia de $24 \%$ y disminuyó al $12 \%$, en 1980 y Giardia lamblia, al contrario de los parásitos anteriormente nombrados, se comportó de manera ascendente en el tiempo, aumentando su prevalencia de $9,4 \%$, reportada en 1965 , a un $21,4 \%$, en 1980 (Corredor E Arciniegas, 2002).

Los jardines infantiles constituyen un problema importante como focos endémicos, ya que los niños infectados pueden transmitir los parásitos a los padres y a otros miembros de la familia, contribuyendo a mantener una alta endemicidad en la comunidad (Mendoza et al. 2001; Nuñez, 2003).

El Distrito Capital de Bogotá está organizado, en su parte administrativa, en 20 localidades. La localidad Tunjuelito representa el 1,2\% del área total de la ciudad, donde predomina la clase media - baja: el 50,2\% de los predios son de estrato tres y el $49,5 \%$ del dos. Esta localidad 226 mil habitantes (3,3\% del total de la ciudad) (Campo, 2007).

Tunjuelito es la novena localidad de Bogotá, con mayor número de personas, 20.965, con necesidades básicas insatisfechas (NBI). El 31,8\% de la población está clasificada en nivel 1 y 2 del SISBEN y el 72,8\% está afiliada al sistema de salud; corresponde a la décimo quinta localidad en cobertura de seguridad social en salud. La mayoría de la población afiliada pertenece al régimen contributivo (77\%) (Saldias, 2003; Campo, 2007).

Como se explicó anteriormente existen condiciones desfavorables para la población afectada, que incrementan el riesgo de estas personas de enfermar y morir, debido a las malas condiciones de salud y de vivienda, por lo tanto, es necesario determinar la prevalencia específica de enteroparasitosis, con el fin de visualizar un panorama localizado de la situación y así proponer las correspondientes acciones de salud pública y de atención médica, dirigida a contrarrestar los diferentes puntos críticos.

\section{MATERIALES Y MÉTODOS}

Se realizó un estudio de tipo observacional descriptivo de corte transversal, con una población de niños y de 
niñas en edad preescolar (2 a 5 años), quienes asistían a jardines seleccionados a orillas del río Tunjuelito, durante agosto y septiembre de 2009.

Para el cálculo del tamaño muestral, se planteó la siguiente fórmula (Fuentelsaz, 2004):

$$
n=\frac{N * Z_{a}^{2} p^{*} q}{d^{2} *(N-1)+Z_{a}^{2} * p^{*} q}
$$

Donde:

1. $\mathrm{N}=$ total de la población (226.000)

2. $\mathrm{Z}_{\mathrm{a}}^{2}=1.96^{2}$ (si la seguridad es del $95 \%$ )

3. $\mathrm{p}=$ proporción esperada (en este caso $28 \%=0,28$ )

4. $\mathrm{q}=1-\mathrm{p}$ (en este caso $1-0,28=0,72)$

5. $\mathrm{d}=$ precisión (en este caso deseamos un $3 \%$ )

- La localidad de Tunjuelito tiene 226.000 habitantes

$$
n=\frac{226000 \times 1.96^{2} \times 0.28 \times 0.72}{0.03^{2}(226000-1)+1.96^{2} \times 0.28 \times 0.72}=857
$$

ajustando el tamaño de la muestra y teniendo en cuenta la cantidad de jardines infantiles y el número de niños y niñas por jardín, tendríamos:

$$
\begin{aligned}
& \mathrm{n} \text { ajustada }=\frac{\mathrm{n}}{1+\mathrm{n} / \mathrm{N}(\text { número de la población })} \\
& \mathrm{n} \text { ajustada }=\frac{857}{1+\frac{857}{105}}=92
\end{aligned}
$$

Lo que implica que la muestra probabilística correspondió a 92 niños (as).

La metodología empleada consistió en proponer y obtener de los padres de familia, de cada uno de los niños seleccionados, el consentimiento de participación y el visto bueno del instrumento que evaluó las variables demográficas y socio-económicas; posteriormente, se entregó a la madre de cada niño tres frascos de plástico estériles, con tapa a presión y sin solución preservante. Las muestras fueron tomadas por defecación espontánea, para lo cual, se explicó a todos los padres de familia las recomendaciones para la correcta toma. Las muestras fueron transportadas al laboratorio de Microbiología de la Universidad de Ciencias Aplicadas y Ambientales U.D.C.A, para su respectivo análisis.

Para el estudio coprológico, se recolectó por cada paciente, tres muestras de heces, obtenidas por evacuación espontánea, en días diferentes. El análisis de las heces, se efectuó mediante un examen directo en solución salina fisiológica y coloración temporal con lugol (Tello E Canales, 2000; Londoño, et al. 2009).

Se realizó y se aplicó a los padres de familia el instrumento que evalúa las variables demográficas y socio-económicas, teniendo en cuenta las variables de impacto relacionadas con las parasitosis intestinales (Cuadro 1).

Los criterios de inclusión y de exclusión de los niños (as), se aprecian en la tabla 1.

Tabla 1. Criterios de inclusión y exclusión del estudio.

\begin{tabular}{|c|c|}
\hline CRITERIOS DE INCLUSIÓN & CRITERIOS DE EXCLUSIÓN \\
\hline Niños y niñas entre los 2 y 5 años de edad & $\begin{array}{c}\text { Niños que hayan vivido menos de dos años en el barrio } \\
\text { Tunjuelito }\end{array}$ \\
\hline Residente del barrio Tunjuelito durante al menos 2 años & $\begin{array}{c}\text { Que hayan recibido tratamiento antiparasitario un mes antes del } \\
\text { estudio }\end{array}$ \\
\hline Que haya estado expuesto a la contaminación del río Tunjuelito & Rechazo a participar del estudio \\
\hline Que desee participar voluntariamente & \\
\hline Que el acudiente firme el consentimiento informado & \\
\hline
\end{tabular}


FACTORES DE RIESG O PARA ENTEROPARASITOSIS

DATOS PERSONALES

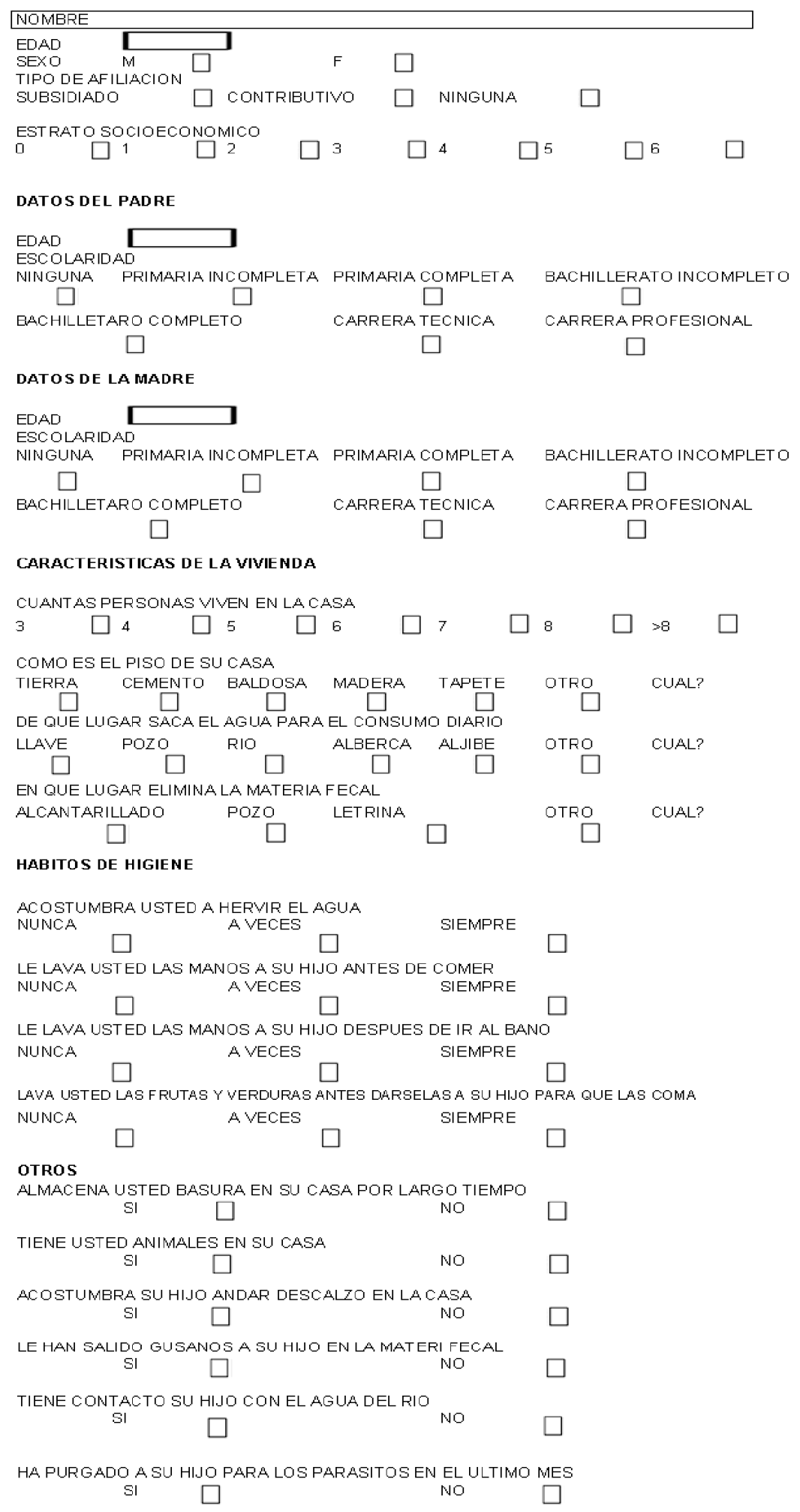

Cuadro 1. Instrumento que evalúa las variables demográficas y socioeconómicas. 
Análisis de los resultados: Los datos fueron tabulados en una hoja de Excel para su respectivo análisis, con la ayuda del software SPSS: Análisis uni y bi-variado. Se aplicó una prueba de Chi cuadrado, para evaluar la diferencia entre el contacto con el agua del río y el resultado positivo del coprológico para parásitos intestinales no patógenos o comensales y también para evaluar la diferencia entre el contacto con el agua del río y el resultado positivo del coprológico para entero parásitos patógenos; además, se practicó una prueba de ANOVA de un factor, para estimar la diferencia entre el estrato socio-económico y el resultado positivo para entero parásitos.

\section{RESULTADOS Y DISCUSIÓN}

La frecuencia de entero parasitosis encontrada en el presente estudio fue del $75 \%$, resultado equivalente en comparación con el estudio llevado a cabo por Agudelo et al. (2008) en un corregimiento de la costa Caribe colombiana, quienes encontraron una frecuencia de enteroparásitos del $92 \%$. En otro estudio realizado en una comunidad Amerindia de Costa Rica, por Hernández $\mathcal{E}$ Matamoros (2005), se halló una prevalencia del 84\%, a diferencia del estudio realizado por Cortés et al. (1999) en niños preescolares institucionalizados en Bogotá, donde se determinó una prevalencia del 19,4\%. La investigación llevada a cabo por Milano et al. (2007) mostró una similaridad con el actual, ya que detectó una prevalencia de niños parasitados de $73,5 \%$.

Teniendo en cuenta los coprológicos positivos, el 17,4\% presentó parásitos intestinales patógenos, como fueron G. lamblia (7,6\%), E. histolytica $(7,6 \%)$ y A. lumbricoides (2,2\%). Chaves et al. (2007) encontraron que la prevalencia de la infección por enteroparásitos, como G. lamblia, no ha cambiado significativamente y continua siendo un problema de salud pública en amplias regiones del mundo y en Colombia, asociándose con un incremento de la pobreza, un saneamiento ambiental inadecuado y deficientes servicios públicos. Por otra parte, Rivera et al. (2008), en contraste con nuestros resultados, al evaluar heces seriadas, mediante examen directo a 47 niños de 1 a 4 años de edad, hallaron una prevalencia de $G$. lamblia de 39,1\% y A. lumbricoides de 21,7 \%; así mismo, reportaron la presencia de factores desfavorables, como la contaminación fecal del agua de consumo y de los alimentos, además de insuficientes condiciones sanitarias y socio-culturales.
Dentro de los parásitos intestinales comensales fueron positivos un $57,6 \%$ correspondientes a: $B$. hominis (60\%), E. nana (30\%), E. coli (8\%), I. butschlii (2\%). Esta prevalencia de protozoarios comensales carece de importancia clínica, pero tiene importancia epidemiológica, pues es referente de la contaminación con materia fecal de los alimentos y del agua de consumo, siendo éstos, los mismos vehículos para la transmisión de otros protozoarios, potencialmente patógenos, lo que concuerda con investigaciones como la realizada por Rivera et al. (2008) y Agudelo et al. (2008), quienes reportaron una prevalencia del $60 \%$ para parásitos comensales. Así mismo, el estudio de Devera (1998) realizado en una comunidad del estado Anzoategui, Venezuela, encontró un 66,7\% de parásitos, contrastando con el trabajo ejecutado por Londoño et al. (2009), en el que se halló $B$. hominis en un $36,4 \%$. Este último es reconocido como uno de los parásitos más prevalentes en diversas partes del mundo (30-50\%) (Zerpa ETerashima, 2000).

Del total de coprológicos positivos, el 23,9\% presentaron una única especie de parásitos, es decir, un monoparasitismo y un $51,1 \%$ mostraron dos o más especies de parásitos, es decir, poli-parasitismo, lo que contrasta con lo hallado por Rivera et al. (2008), quienes registraron un $69,6 \%$ para monoparasitismo y $30,4 \%$ de poliparasitismo.

Fueron cinco variables las que manifestaron concordancia con el resultado parasitario. La variable de contacto con el agua del río se relacionó con el resultado coprológico, tanto para entero parásitos patógenos $(p=0,000)$ como para los no patógenos $(p=0,000)$, posiblemente, dado por la contaminación de las aguas lluvias propias de la cuenca del río Tunjuelito con las aguas negras de los barrios aledaños que llegan a éste, lo que favorece la persistencia de los ciclos vitales de estos microorganismos. 35,9\% de la población estudiada había tenido algún contacto con el agua del río Tunjuelito y un $64,1 \%$ no refirió contacto, lo que indica que más de un tercio de la población estudiada revela un importante factor de riesgo de infección parasitaria, lo cual, es concordante con Rivera et al. (2008), quienes encontraron la presencia de agentes desfavorables, como la contaminación fecal del agua de consumo de los alimentos, además de deficientes condiciones sanitarias y socio-culturales. 
Se observó que un 96,7\% de las viviendas de la población estudiada obtenía el agua a partir del acueducto y un 3,3\% lo hacía a partir de una alberca. Se observó que el $100 \%$ de la población estudiada eliminaban las excretas a través del alcantarillad; esto hace que no sea significativa la variable, debido a la buena obtención de este recurso natural y a la buena eliminación de excretas.

El estrato socio-económico bajo, se relacionó con un resultado coprológico positivo para parásitos intestinales patológicos $(p=0,48)$, posiblemente, por las condiciones de vida, de saneamiento, por la falta de prácticas saludables de higiene, desnutrición asociada y otros factores propios de poblaciones, con estrato socioeconómico bajo. El 76,1\% pertenecían al estrato uno, el $19,6 \%$ al estrato dos y el $4,3 \%$ a estrato cero, lo que es concordante con la distribución de la población en la localidad de Tunjuelito, donde predominan los estratos uno y dos.

Las características del piso de la vivienda, se relacionaron con el resultado coprológico positivo para parásitos intestinales patógenos $(p=0,001)$. En el $63 \%$ de las viviendas de la población estudiada el piso era en cemento, $26,1 \%$ en baldosa, $7,6 \%$ en tierra y $3,3 \%$ en madera.

El29,3\% de la población almacenaba la basura por periodos prolongados y un $70,7 \%$ no lo hacían, encontrándose diferencias, estadísticamente significativas, $(0,046)$, lo cual, indica una falta de higiene en casi un tercio de la población estudiada, aumentando así el riesgo de infecciones gastrointestinales parasitarias.

El caminar descalzo, se relacionó con el resultado coprológico positivo para parásitos intestinales patógenos $(p=0.041)$, ya que este hábito tiene como consecuencia la puerta de entrada para la infección por geo-helmintos, aunque, probablemente, existan otros factores más importantes asociadas a ésta variable, ya que la prevalencia en este estudio de geo-helmintos fue muy baja. Se observó que un 45,7\% tenían como hábito deambular descalzo en la casa y un $54,3 \%$ no lo tenía. Se demostró un incremento en la presencia de entero parasitosis, como lo revela también el estudio de parásitos intestinales en una comunidad aborigen de la provincia de Salta, Buenos Aires, Argentina, por Menghi (2007) y el trabajo reportado por Milano et al. (2007), quienes descubrieron en su estudio de entero parasitosis que el hábito de deambular descalzos, de jugar en el suelo y las deficiencias en el manejo de excretas conformaron el escenario óptimo para la transmisión de los geo-helmintos diagnosticados

Del total de encuestados (92), el 56,52\% eran niños y el $43,5 \%$ eran niñas. Los padres autorizaron la participación en el estudio y respondieron el instrumento que evaluaba los factores de riesgo demográficos y socioeconómicos.

En la población estudiada, se observó que un $84,8 \%$ pertenecían al régimen subsidiado, un $15,2 \%$ no tenía afiliación al régimen de seguridad social en salud y ninguno pertenecía al nivel contributivo. Lo que se interpretaría como que un gran porcentaje de la población analizada presenta dificultades para el acceso a los servicios de salud, convirtiéndolos más vulnerables en presencia y persistencia de parasitismo intestinal; estos datos son concordantes con los reportados por Chaves et al. (2007), quienes sugieren en su trabajo, sobre factores asociados a la infección por G. duodenalis en escolares y preescolares de una zona rural de Cundinamarca, que el pertenecer al régimen subsidiado, se asocia con la infección por enteroparásitos.

El análisis socio-demográfico muestra que un gran porcentaje de la población estudiada presenta dificultades para el acceso a los servicios de salud por estar como subsidiados dentro del sistema general de seguridad social en salud, concordando con Sánchez et al. (2000) en el estudio de asentamientos humanos irregulares en México. En la comunidad analizada, el estrato uno predominó y hay un significativo registro, de un $19 \%$, donde las madres finalizaron la educación secundaria, mientras que un $15,2 \%$ de los padres no lo hizo, mostrando que existe un alto porcentaje de estudios básicos incompletos, lo cual, también fue demostrado por Londoño et al. (2009). Todo esto hace que se incremente la vulnerabilidad en presencia y en persistencia de parasitismo intestinal, ajustándose a lo expuesto en el estudio llevado a cabo por Soriano et al. (2005), en la Patagonia, Argentina.

Se advirtió que la edad promedio del padre fue de 29 años, la mediana de 29 y la moda de 29, lo que representa una distribución normal; se percibió que la edad mínima fue de 19 años y la edad máxima 46 años. La edad promedio de la madre fue de 26 años, 
la mediana de 26 y la moda de 26, lo cual, representa una distribución normal. Se observó que la mínima edad fue de 17 años y la edad máxima 40, indicando una media poblacional joven de los padres que influye en la escolaridad incompleta.

El 30,4\% de los padres (hombres) poseían estudios de bachillerato incompleto; un $27,2 \%$ tenían la primaria completa y un $25 \%$ incompleta; $15,2 \%$ habían completado el bachillerato, un 1,1\% cursó una carrera técnica y un $1,1 \%$ no tenían estudios. El 33,7\% de las madres habían terminado la primaria; un 23,9\% tenían el bachillerato incompleto y un $19 \%$ el completo; un $18,5 \%$ tenían primaria incompleta y un $4,3 \%$ poseía una carrera técnica. Basados en lo anterior, todos los padres de los niños poseían algún grado de estudio, donde se podría analizar que conocen las medidas básicas para evitar un contagio por parásitos.

Respecto a los hábitos de higiene personal, como lavarse las manos antes de comer y después de ir al baño, hervir el agua antes de consumirla y el lavado de frutas, se encontró que un alto porcentaje de la población no los practicaba, similar con el estudio de Ibáñez et al. (2004), quienes evaluaron el entero parasitismo en escolares de comunidades nativas del alto marañón, Amazonas, Perú. En la población estudiada, se observó que un 91,3\% no tenían como hábito lavar las frutas y verduras para su consumo y un $8,7 \%$, sí. Estas variables son significativas, ya que se podría afirmar que, debido al no hervir el agua y no lavar adecuadamente los alimentos, hay más posibilidad de adquirir entero-parásitos.

El 96,7\% de la población no tenían como hábito lavarse las manos antes de consumir alimentos y un 3,3\% sí lo poseían. El 95,7\% carecía del hábito de lavarse las manos después de ir al baño y un 4,3\%, sí. De acuerdo con lo anterior, el no realizar frecuentemente el lavado de manos sabiendo que la principal vía de transmisión parasitaria es mano - boca, aumenta la prevalencia de adquirir entero-parásitos.

La presencia de fauna doméstica fue del $48,9 \%$ teniendo uno o más animales, semejante al reportado en el estudio de prevalencia de parásitos intestinales en escolares de primaria de Santiago de Surco, Lima, Perú, por Iannacone et al. (2006). En cambio Milano et al. (2007) encontraron que en el 95,5\% de las unidades domésticas contenían uno o más perros y gatos, resaltando, en sus conclusiones, que se debe insistir, simultáneamente al tratamiento farmacológico, en las medidas preventivas relacionadas con la higiene y la adecuada eliminación de las excretas humanas y de los animales domésticos.

De la población evaluada, el 1,1\% refirió haber expulsado una forma adulta de helmintos en materia fecal y un 98,9\% negó esta pregunta, para lo cual, se podría argumentar que hay una baja prevalencia de helmintos en la población estudiada, ya que una vez dichos organismos cumplen su ciclo vital, van al exterior.

El análisis del presente estudio mostró que había cinco habitantes en la casa (43,5\%), haciendo de esto un factor predisponente para la diseminación de parasitismo en círculos cerrados; además, el 7,6\% de estas viviendas el piso era en tierra. Se notó que habían buenos niveles de acceso a servicios públicos, agua y eliminación de excretas, lo que concuerda con el estudio divulgado por Fernández et al. (2007), realizado en La Virgen, Cundinamarca.

Milano et al. (2007) argumentaron una vez más que la aplicación del concepto eco-sistémico al campo de la salud humana, requiere no sólo de la identificación del agente etiológico de la enfermedad, sino también del conjunto de determinantes genéticos, biológicos y socio-económicos, que configuran los niveles de salud y enfermedad de una población.

Todos los niños y niñas quienes obtuvieron un resultado positivo para parásitos intestinales, se remitieron a su respectivo servicio de salud, con un certificado del análisis coprológico, concluyendo que existen condiciones en la población estudiada, que constituyen un contexto favorable para la elevada frecuencia de las enfermedades parasitarias intestinales.

AGRADECIMIENTOS: Los autores agradecen la colaboración prestada a la Universidad de Ciencias Aplicadas y Ambientales U.D.C.A, directivas y docentes de la facultad de Medicina, así mismo a los Jardines infantiles, rectoras, docentes, padres de familia y niños participantes en el desarrollo de esta investigación. Conflicto de intereses: Los autores del presente artículo declaramos que no existen conflictos de intereses que pongan en riesgo la validez de los resultados presentados. Financiación: El trabajo fue financiado por los autores del presente trabajo en colaboración con el laboratorio 
de Microbiología de la Universidad de Ciencias Aplicadas y Ambientales U.D.C.A.

\section{BIBLIOGRAFÍA}

1. AGUDELO, S.; GÓMEZ, L.; CORONADO, X.; OROZCO, A.; VALENCIA, C.; RESTREPO, L.; GALVIS, L.; BOTERO, L. 2008. Prevalencia de Parásitos intestinales y factores asociados en un corregimiento de la costa Atlántica. Rev. Salud Pública, Colombia. 10(4):633-642.

2. BOTERO, D.; RESTREPO, M. 2003. Parasitosis humanas. Técnicas de laboratorio en parasitología medica. Corporación para investigaciones biológicas. Medellín, Colombia. p.455-483.

3. CORREDOR, A.; ARCINIEGAS, E. 2002. Parasitismo Intestinal. Instituto Nacional de Salud. Santafé de Bogotá. p.90.

4. CAMPO, M. 2007. Pérfil económico y empresarial en la localidad de Tunjuelito. Cámara de Comercio de Bogota. LEGIS SA. p.74.

5. CHÁVES, M.; FERNANDEZ, J.; OSPINA, I.; LÓPEZ, M.; MONCADA, L.; REYES, P. 2007. Tendencia de la prevalencia y factores asociados a la infección por Giardia duodenalis en escolares y preescolares de una zona rural de Cundinamarca. Revista Biomédica 27:345-351.

6. CORTÉS, J.; SALAMANCA, L.; SÁNCHEZ, M.; VANEGAS, F.; SIERRA, P. 1999. Parasitismo y estado nutricional en niños preescolares de instituciones de Santafé de Bogotá. Pediatría (Bogotá), 34(4):288-291.

7. DEVERA, R. 1998. Blastocystis hominis: o enigma continúa. Rev. Soc. Bras. Med. Trop. 31(5):491492.

8. FERNÁNDEZ, J.; REYES, P.; MONCADA, L.; LÓPEZ, M.; CHÁVES, M.; KNUDSON, A.; ARIZA, Y. 2007. Tendencia y prevalencia de las geohelmiantiasis en la Virgen, Colombia 1995-2005. Revista Salud Pública. 9(2):289-296.
9. FUENTELSAZ, G. 2004. Cálculo del tamaño de la muestra. Matronas Profesión. (España). 5(18): 5-13.

10. HERNÁNDEZ, F.; MATAMOROS, M. 2005. Parásitos intestinales en una comunidad Amerindia, Costa Rica. Revista Parasitol. Latinoam. 60:182-185.

11. IBÁÑEZ, N.; JARA, C.; GUERRA, A.; DÍAZ, E. 2004. Prevalencia del Enteroparasitismo en escolares de comunidades nativas del Alto Marañón, Amazonas, Perú. Rev. Peruana Med. Exp. y Salud Pública. 21(3):126-133.

12. IANNACONE, J.; BENITES, M.; CHIRINOS, L. 2006. Prevalencia de infección por parásitos intestinales en escolares de primaria de Santiago de Surco, Lima, Perú. Revista Parasitol. Latinoam. 61:5462.

13. LONDOÑO, Á.; MEJIA, S.; GÓMEZ, J. 2009. Prevalencia y factores de riesgo asociados a Parasitismo Intestinal en Preescolares de Zona Urbana en Calarcá, Colombia. Revista Salud Publica. 11(1):72-81.

14. MARCOS, L.; MACO, V.; TERASHIMA, A.; SAMAIVIDES, F.; GOTUZZO, E. 2003. Parasitosis intestinal en poblaciones urbanas y rural en Sandia, departamento Puno, Perú. Revista Parásitol. Latinoam. 58:35-40.

15. MENDOZA, D.; NUÑEZ, F.; ESCOBEDO, A.; PELAYO, L.; FERNÁDEZ, M. 2001. Parasitosis intestinales en 4 círculos infantiles de San Miguel del Padrón, ciudad de la Habana, 1998. Rev. Cubana Med. Trop. 53(3):189-193.

16. MENGHI, C.; IUVARO, F.; DELLACASA, M.; GATTA, C. 2007. Investigación de parásitos intestinales en una comunidad aborigen de la provincia de Salta. (Buenos Aires - Argentina). Revista de Medicina. 67(6):705-708.

17. MILANO, A.; OSCHEROV, E.; PALLADINO, A.; BAR, A. 2007. Enteroparasitosis infantil en un área urbana del nordeste argentino. Medicina (Argentina). 67:238-242. 
18. MORRONE, F., CARNEIRO, J., REIS,C. 2004. Study of enteroparasites infection frequency and chemotherapeutic patients in a community living in Porto Alegre,Rs, Brazil. Rev Inst Med Trop S Paulo 46(2):77-80.

19. NUÑEZ, F. 2003. Factores de riesgo de la infección por Giardia lamblia en niños de guarderías infantiles de ciudad de la Habana, Cuba. Cad. Saúde Pública, Rio de Janeiro. 19(2):677-682.

20. RIVERA, M.; LÓPEZ, J.; RODRÍGUEZ, C. 2008. Enteroparasitosis infantil en guarderias de la zona rural de Cajamarca. Rev. Peru. Med. Exp. Salud Pública. 25(4):445-445.

21. SALDIAS, C. 2003. Monografia localidad de Tunjuelito. Departamento Administrativo de Planeación Distrital. Alcaldia Mayor de Bogota D.C. 49p.

22. SÁNCHEZ, J.; TAY, J.; ROBERT, L.; ROMERO, R.; RUÍZ, D.; RIVAS, C. 2000. Frecuencia de Parásitos intestinales en asentamientos humanos irregulares. Rev. Fac. Med. UNAM, México. 43(3):80-83.
23. SAVIOLI, L.; BUNDY, D.; TOMKINS, A. 1992. Intestinal parasitic infections: a soluble public health problem. Trans R. Soc, Trop. Med. Hyg. 86:353354.

24. SORIANO, S.; MANACORDA, A.; PIERANGELI, N.; NAVARRO, M.; GIAYETTO, A.; BARBIERI, L.; LAZZARINI, L.; MINVIELLE, M.; GRENOVERO, M.; BASUALDO, J. 2005. Parasitosis intestinales y su relación con factores socioeconómicos y condiciones de hábitat en niños de Neuquén, Patagonia, Argentina. Parasitol. Latinoam. 60:154-161.

25. TELlO, R.; CANALES, M. 2000. Técnicas de diagnóstico de enfermedades causadas por enteroparásitos. Diagnóstico (Perú). 39(4):197-198.

26. ZERPA, R.; TERASHIMA, A. 2000. Blastocystosis. Diagnóstico. 39(3):120-121

Recibido: Enero 18 de 2010

Aceptado: Mayo 4 de 2010 